\title{
Clear Cell Papillary Renal Cell Carcinoma: A Rare Subtype
}

\author{
Armin Kamyab, Michael J. Jacobs
}

Department of Surgery, Providence Hospital and Medical Centers, Southfield, USA.

E-mail: mjjacobs@pol.net

Received August 26 $6^{\text {th }}, 2011$; revised September $30^{\text {th }}$, 2011; accepted October $9^{\text {th }}, 2011$.

\begin{abstract}
Introduction: Renal cell carcinoma (RCC) is generally divided into clear cell, papillary, and chromophobe subtypes. Clear cell papillary RCC is a recently described subtype. Case report: We report the case of a 42 year old female who was found on computed tomography scan of the abdomen to have a cyst which appeared to involve the right kidney. Pathology of the cyst wall revealed features consistent with a RCC, clear cell papillary type. Discussion: Clear cell papillary RCC are a recently described entity with less than 100 cases reported in the literature. Most cases in the literature have been associated with end-stage renal disease, however more case are being reported in patients without renal disease. Conclusion: Clear cell papillary RCC is a rare distinct entity in the spectrum of renal cell carcinoma. Based on the literature they appear to be clinically indolent tumors; however, more research is required to further characterize and prognosticate these rare tumors.
\end{abstract}

Keywords: Renal Cell Carcinoma, Clear Cell, Papillary

\section{Introduction}

Renal cell carcinomas (RCCs) are usually solid renal masses, but may also present as cystic lesions. They are generally divided into clear cell, papillary, and chromophobe subtypes. Clear cell papillary renal cell carcinomas are a recently described entity with very few cases reported in the literature.

Most cases in the literature have been associated with end-stage renal disease; however, more cases are being reported in patients without renal disease. We describe a case of a clear cell papillary RCC in a 42 year-old female.

\section{Case Report}

We report the case of a 42 years old female who had been having recurring right-sided abdominal pain associated with diarrhea. The patient had an extensive history of endometriosis. She had no other medical history, and was not taking any medications. During work up she underwent a computed tomography (CT) scan which revealed a simple appearing $6 \times 6 \mathrm{~cm}$ cyst which appeared to involve the right kidney. The patient underwent a follow up CT scan 6 months later, which again demonstrated a stable $6 \times 6 \mathrm{~cm}$ simple cyst thought to be arising from the right kidney (Figures 1 and 2).
Since the patient was symptomatic, the decision was made for percutaneous aspiration of the cyst for pathologic examination. Prior to the aspiration, the patient underwent an ultrasound evaluation, which revealed a simple $7.8 \mathrm{~cm}$ cyst with a $10 \mathrm{~mm}$ echogenic excrescence (Figure 3). This was considered Bosniak IIF (Table 1). The patient subsequently underwent a CT-guided percutaneous aspiration of the cyst. Approximately $210 \mathrm{~mL}$ of dark-colored fluid was aspirated and sent to the lab. Post procedure imaging demonstrated resolution of the cyst.

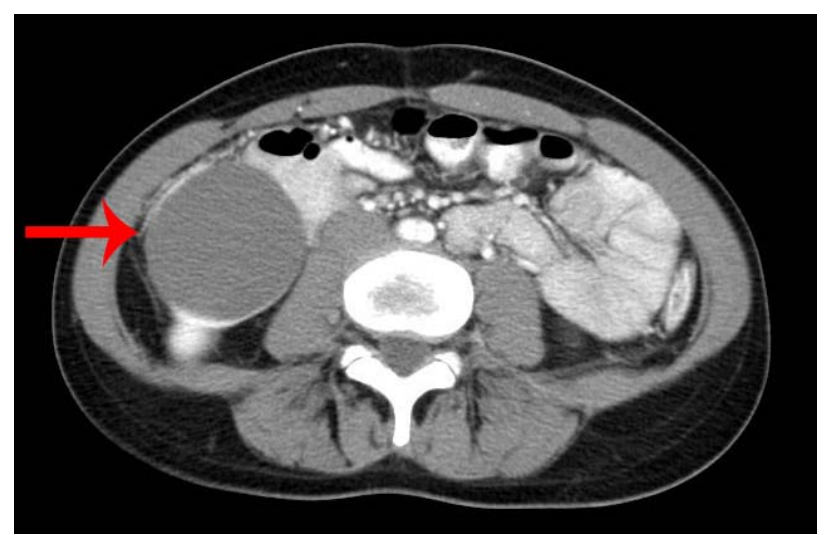

Figure 1. Axial CT scan demonstrating a large cystic lesion (red arrow). 


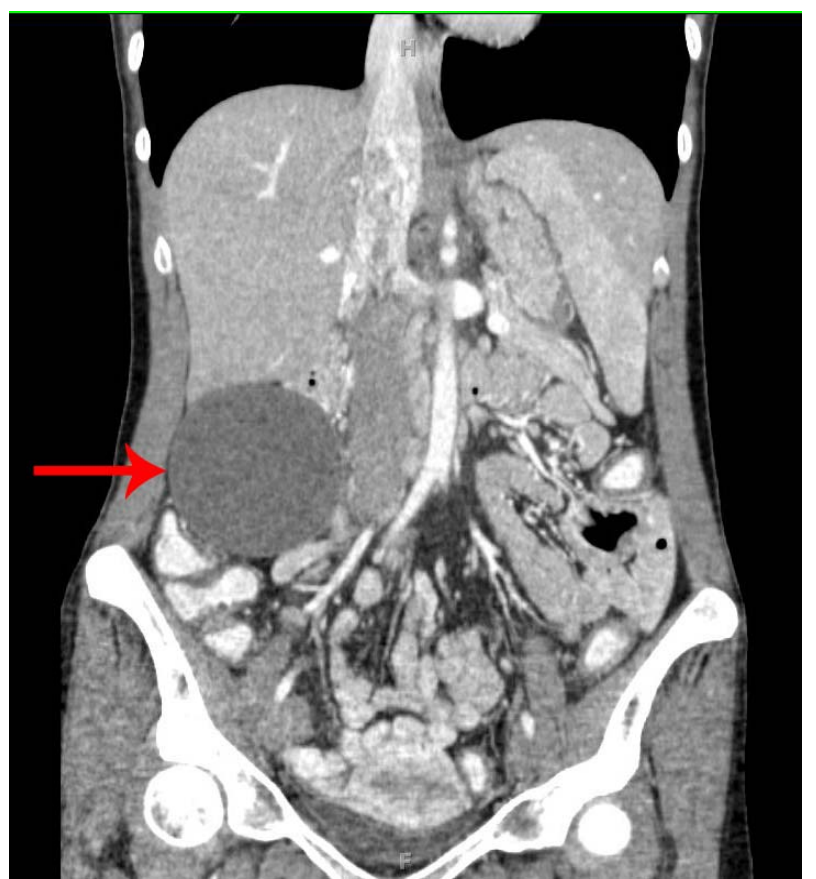

Figure 2. Coronal CT scan demonstrating a large cystic lesion (red arrow).

Following the percutaneous aspiration, the patient's symptoms initially resolved. The pathologic evaluation of the aspirate revealed dark colored fluid, with atypical cells, without any histologically noticeable pathology. The cyst and her symptoms however recurred shortly thereafter and the patient was therefore referred to the surgeon for definitive therapy.

Decision was made for a diagnostic laparoscopy, with laparoscopic cyst aspiration, and excision of the cyst wall for pathological evaluation. Intraoperatively, a mobile dominant cyst in the right upper quadrant was noted. This appeared to be adjacent to the right kidney, the transverse colon, and the duodenum. Serosanguinous fluid was aspirated, and the cyst was subsequently marsupialized. A cherry red exophytic nodule, which corresponded to the Bosniak IIF echogenic excrescence seen on ultrasound, was noted on the luminal side of the cyst wall. An excisional biopsy of this lesion was performed.

The patient tolerated the procedure well and was discharged the morning after surgery. Pathology of the cyst wall revealed a fibrous walled cyst with a thin rim of renal parenchyma with atrophic changes and hyalinization. On some sections, there were nodules of tumor composed of cells with clear cytoplasm arranged in tubules, with few of the tubules having papillary projections without any true fibrovascular core. The nuclei in these cells were linearly arranged and polarized away from the basement membrane. Immunostains showed that the cells were strongly positive for cytokeratin 7 , cytokeratin AE1/AE3, EMA, Vimentin, and negative for CD10 and cytokeratin 20. The immunomorphologic features were consistent with a RCC, clear cell papillary type.

\section{Discussion}

Renal cell carcinoma is generally divided into clear cell, papillary, and chromophobe subtypes [1]. Each has characteristic cytogenetic aberrations that can aid in their classification. Clear cell carcinoma is associated with loss of 3p, papillary carcinoma is associated with trisomy 7,16 , and 17 , and chromophobe carcinoma is associated with hypodiploid with loss of chromosomes $1,2,6,10$, 13,17 , and 21 [2].

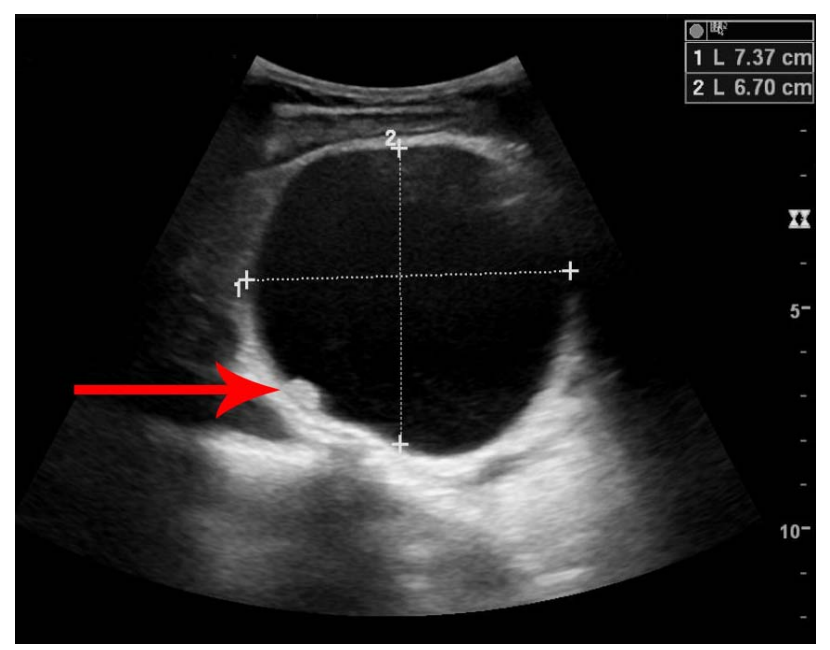

Figure 3. Ultrasound demonstrating a simple cyst with an echogenic excrescence (red arrow).

Table 1. Bosniak classification system [3].

Category I: simple benign cysts showing homogeneity, water content, and a sharp interface with adjacent renal parenchyma, with no wall thickening, calcification, or enhancement.

Category II: cystic lesions with $<1 \mathrm{~mm}$ thick septations or thin, fine calcification in their walls or septa, $<3 \mathrm{~cm}$ in diameter, and hyperdense cysts.

Category IIF: cystic lesions with multiple thin septum, calcification, $>3 \mathrm{~cm}$, and lack of contrast enhancement.

Category III: cystic lesions with uniform wall thickening, thick/irregular calcification, thick septa, and contrast enhancement.

Category IV: lesions with a non-uniform or enhancing thick wall, enhancing or large nodules in the wall, or clearly solid components in the cystic lesion. 
Although most RCCs are solid renal masses, they may also be cystic lesions. To differentiate between benign and malignant cystic renal lesions, the Bosniak classification system was devised to classify cysts into four different categories based on their radiological features [3] (Table 1). In our patient, an echogenic excrescence was noted within the cyst, therefore classifying it as a Bosniak category IIF cyst.

Clear cell papillary RCCs are a recently described entity with less than 100 cases reported in the literature. They are a group of renal tumors composed of cells with clear cytoplasms arranged in papillary patterns [4]. They were initially reported in the setting of end-stage renal disease, but are now more commonly reported in the setting of non-end-stage renal disease.

The cytogenetic and immunohistochemical phenotypes of these unusual renal tumors have been studied [5]. It was demonstrated that these tumors lacked the gain of chromosome 7 and loss of chromosome $\mathrm{Y}$ that is typical of papillary RCC [4]. Deletion of chromosome 3p, which is usually seen in clear cell RCC was not detected [4]. The authors concluded that the genetic and immunophenotypic profile of these tumors were distinct from those of either papillary or clear cell RCC, and therefore 'clear cell papillary RCC' is a distinct entity in the spectrum of RCC [4].

Clear cell papillary RCC usually presents with low pathological stage and appears to be clinically indolent [6]. A recent review of 40 cases with a mean follow-up period of 28 months showed no evidence of disease after treatment [6]. More research is required to further characterize and adequately prognosticate these newly described tumors.

\section{Conclusions}

Clear cell papillary RCC is a distinct entity in the spectrum of RCC. Most cases in the literature have been associated with end-stage renal disease; however, more cases are being reported in patients without renal disease. Based on the literature they appear to be clinically indolent tumors; however, more research is required to further characterize and prognosticate these newly described tumors.

\section{REFERENCES}

[1] V. E. Reuter and J. C. Presti, "Contemporary Approach to the Classification of Renal Epithelial Tumors,” Seminars in Oncology, Vol. 27, No. 2, 2000, pp. 124-137.

[2] E. Van Den Berg and S. Storkel, "Kidney: Renal Cell Carcinoma,” Atlas Genet Cytogenet Oncol Haematol, June 2003.

[3] M. A. Bosniak, "The Current Radiological Approach to Renal Cysts,” Radio, Vol. 158, No. 1, 1986, pp. 1-10.

[4] S. Gobbo, J. M. Eble, D. J. Grignon, et al., "Clear Cell Papillary Renal Cell Carcinoma: A Distinct Histopathologic and Molecular Genetic Entity," American Journal of Surgical Pathology, Vol. 32, No. 8, 2008, pp. 1239-1245. doi:10.1097/PAS.0b013e318164bcbb

[5] Z. Bing and J. Tomaszewski, "Clear Cell Papillary Renal Cell Carcinoma in the Bilateral Native Kidneys after 2 Years of Renal Transplantation: Report of a Case and Review of the Literature," Case Reports in Transplantation, Vol. 2011, 2011, Article ID 387645, 4 pages. doi: $10.1155 / 2011 / 387645$

[6] H. Aydin, L. Chen, L. Cheng, et al., "Clear Cell Tubulopapillary Renal Cell Carcinoma: A Study of 36 Distinctive Low-Grade Epithelial Tumors of the Kidney," American Journal of Surgical Pathology, Vol. 34, No. 11, 2010, pp. 1608-1621. 